\title{
Progressive subcortical gliosis and progressive supranuclear palsy can have similar clinical and PET abnormalities
}

Norman L Foster, Sid Gilman, Stanley Berent, Anders A F Sima, Constance D'Amato, Robert A Koeppe, Samuel P Hicks

\begin{abstract}
In studies of cerebral glucose metabolism utilising positron emission tomography (PET) with ${ }^{18}$ F-fluoro-2-deoxy-D-glucose, patients with the clinical picture of progressive supranuclear palsy (PSP) have shown predominantly frontal glucose hypometabolism. This is presumed to represent deafferentation of cerebral cortical from subcortical structures. In these studies, however, the diagnosis of PSP has not been verified by pathological examination. Necropsy examinations were performed in three patients with the clinical features of PSP in whom PET had demonstrated predominantly frontal hypometabolism. In two of these patients the diagnosis of PSP was confirmed pathologically, and no morphological abnormalities were found in the cerebral cortex. The third patient had extensive cortical and subcortical neuronal loss and gliosis without neurofibrillary tangles, consistent with the diagnosis of progressive subcortical gliosis (PSG). Even in retrospect no unique clinical neurological abnormality or finding on laboratory investigation could be identified that distinguished this latter patient from those with pathologically confirmed PSP. We conclude that PSG and PSP may be indistinguishable during life, and necropsy confirmation is needed for definite diagnosis.
\end{abstract}

University of Michigan Medical Center, Ann Arbor, Michigan, USA Department of

Neurology

N L Foster

$S$ Gilman

Department of

Psychiatry

$S$ Berent ${ }^{\star}$

Division of

Neuropathology

A A F Sima

C D'Amato

S P Hicks

Division of Nuclear

Medicine

R A Koeppe

Correspondence to:

Dr Foster, Department of

Neurology, Taubman Center

$1920 / 0316$, University of

Michigan Medical Center,

1500 East Medical Center

Drive, Ann Arbor, Michigan,

48109-0316,USA

Received 2 January 1991

and in revised form

30 September 1991

Accepted 14 October 1991 posterior temporoparietal hypometabolism is typically seen in Alzheimer's disease. While functional brain imaging may sometimes assist in the differential diagnosis of PSP, significant diagnostic obstacles still exist.

Many neurological disorders cause clinical signs that are similar to those seen in PSP. A downgaze supranuclear gaze palsy, the clinical hallmark of PSP, can occur in disorders as diverse as Whipple's disease, multiple sclerosis, syphilis, brucellosis and Fisher's syndrome. Downgaze supranuclear ophthalmoplegia accompanied by dementia or signs of extrapyramidal disease has been reported in several disorders, such as autosomal dominant olivopontocerebellar atrophy, ${ }^{8}$ diffuse Lewy body disease, ${ }^{9}$ advanced amyotrophic lateral sclerosis, Huntington's disease, Jakob-Creutzfeldt disease, ${ }^{10}$ Niemann-Pick disease type $C,{ }^{11}$ and progressive subcortical gliosis (PSG). ${ }^{12}$ Thus the pitfalls of clinical diagnosis of PSP are substantial. In addition, in one series, 19 of 58 patients with clinical features of PSP had CT or MRI evidence of a multi-infarct state (which may or may not have been responsible for the PSP symptoms). One of these patients at necropsy had multiple small infarcts due to cerebral amyloid angiopathy without pathological features typically found in PSP. ${ }^{13}$ However, this article mentions only the presence of a vertical supranuclear gaze palsy, without specifying whether downgaze was involved. Another paper reporting reversible supranuclear palsy (including downgaze palsy) due to Parkinson's disease is difficult to interpret in the face of a history of concurrent infection in each case, limited information about the patients' clinical course and the absence of pathological data. ${ }^{14}$ Multiple system atrophy (MSA) may also exhibit supranuclear gaze palsy, but it has been proposed that a predominant downgaze paresis should constitute an exclusion criterion for the clinical diagnosis of MSA. ${ }^{15}$

We were able to examine the clinical utility of PET in the differential diagnosis of PSP by reviewing our findings in three patients with the clinical diagnosis of PSP who had participated in a study of FDG-PET ${ }^{2}$ and subsequently died and had postmortem examinations. Although all of these patients had the typical clinical features of PSP, one was subsequently found at necropsy not to have PSP, but rather a multisystem degeneration with a constellation of features best described as PSG. ${ }^{121617}$ This disorder is pathologically distinct from PSP in that there is a complete absence of subcortical neurofibrillary tangles. 


\section{Subjects and methods}

In a previous study ${ }^{2} 14$ patients with typical clinical features of PSP and 21 normal controls of similar age were scanned with FDG-PET and received a standard battery of neuropsychological tests. The selection criteria for these subjects, scanning conditions and technique, are described in our previous report. ${ }^{2}$ For this study we have used the data obtained in our previous study and calculated several additional measures for comparison of individual patients. The most superior transverse slice through the top of the centrum semiovale was further analysed, since cerebral cortical hypometabolism was found to be most affected at this level in our earlier study. Frontal cortex metabolism was determined by computing the mean value of 4 anterior sectors in this slice (regions 2 and 3 in each hemisphere as illustrated in figure 1 of reference 2), and relative frontal metabolism was the ratio of this value to mean overall cerebral cortical metabolism. An anterior to posterior ratio was also calculated (regions 2 and 3/regions 6 and 7 from figure 1 in reference 2), since this ratio has been found useful in distinguishing Alzheimer's disease and Parkinson's disease with dementia from normal subjects and from patients with PSP.5 ${ }^{5}$

Table 1 Clinical features of patients with postmortem examinations

\begin{tabular}{llll}
\hline & $\begin{array}{l}\text { Case 1 } \\
\text { (PSG) }\end{array}$ & $\begin{array}{c}\text { Case } 2 \\
\text { (PSP) }\end{array}$ & $\begin{array}{l}\text { Case 3 } \\
(P S P)\end{array}$ \\
\hline Sex & $\mathrm{M}$ & $\mathrm{F}$ & $\mathrm{F}$ \\
Age at onset (years) & 57 & 58 & 65 \\
Duration of Illness (years) & 4 & 6 & 5 \\
Dementia & ++ & ++ & + \\
Oculomotor abnormality & UGP & UGP & UGP \\
& DGP & DGP & DGP \\
Nuchal rigidity & OCRP & OCRP & OCRP \\
Limb rigidity & ++ & +++ & ++ \\
Increased deep tendon reflexes & ++ & ++ & + \\
Plantars & ++ & +++ & ++ \\
Tremor & $\uparrow \uparrow$ & $\downarrow \downarrow$ & $\downarrow \downarrow$ \\
\hline
\end{tabular}

Abbreviations: PSG = progressive subcortical gliosis, PSP = progressive supranuclear palsy, UGP $=$ upgaze paresis, $\mathrm{DGP}=$ downgaze paresis, OGRP = oculocephalic responses present, $0=$ absent, $+\stackrel{=}{=}$ Mild, $++=$ Moderate, $+++=$ Severe.

Table 2 Glucose metabolism and neuropsychological performance at the time of positron emission tomography scanning

\begin{tabular}{|c|c|c|c|c|c|}
\hline & $\begin{array}{l}\text { Case } 1 \\
\text { (PSG) }\end{array}$ & $\begin{array}{l}\text { Case } 2 \\
(P S P)\end{array}$ & $\begin{array}{l}\text { Case } 3 \\
(P S P)\end{array}$ & $\begin{array}{l}\text { Probable } \\
P S P^{\prime \prime} \\
n=11\end{array}$ & $\begin{array}{l}\text { Normal } \\
\text { Controls } \\
n=21\end{array}$ \\
\hline \multicolumn{6}{|l|}{ Cerebral Cortical Metabolism } \\
\hline Mean cerebral cortex & 4.87 & 4.35 & 5.70 & $4.91(0.51)$ & $6.08(0.94)^{\star}$ \\
\hline Frontal cortex & 3.77 & 4.01 & 5.67 & $4.77(0.35)$ & $6.65(1.11)^{\star \star}$ \\
\hline Caudate nucleus & 5.05 & 4.82 & 5.37 & $5.03(0.71)$ & $6.47(0.91)^{\star \star}$ \\
\hline Putamen & 5.60 & 3.91 & 6.61 & $5.66(0.93)$ & $7.14(1.09)^{\star}$ \\
\hline Thalamus & 4.94 & 4.98 & 5.81 & $5.76(1.27)$ & $6.69(1.29)+$ \\
\hline Pons & 4.37 & 3.44 & 3.58 & $3.81(0.51)$ & $4.45(0.67)^{\star}$ \\
\hline Cerebelar vermis & 5.52 & 4.49 & 5.37 & $5.39(0.92)$ & $5.54(0.86)$ \\
\hline Cerebellar hemisphere & 5.20 & 4.95 & 5.85 & $5.58(0.08)$ & $6.10(0.90)$ \\
\hline Relative frontal hypometabolism & 0.78 & 0.92 & 0.99 & $0.98(0.08)$ & $1.03(0.04)^{\star \star}$ \\
\hline $\begin{array}{l}\text { Anterior to posterior cerebral } \\
\text { cortical glucose metabolism }\end{array}$ & 0.77 & 0.91 & 0.89 & $0.93(0.08)$ & $1.03(0.05)^{\star \star}$ \\
\hline \multicolumn{6}{|l|}{$\begin{array}{l}\text { Cortical glucose metabolism } \\
\text { Neuropsychological performance }\end{array}$} \\
\hline WAIS-R FSIQ & 75 & 75 & 94 & $81(8.2)$ & $117(13.3)^{\star \star}$ \\
\hline WAIS- $R$ vocabulary & 6 & 5 & 10 & $8(2.0)$ & $13(2.5)^{\star \star}$ \\
\hline WAISR Digit Symbol & 3 & 4 & 8 & $5(1.2)$ & $12(2.5)^{\star \star}$ \\
\hline Wechsler MQ & 66 & 83 & 121 & $86(12.5)$ & $129(13.4)^{\star \star}$ \\
\hline Verbal fluency & 1 & 4 & 10 & $4(1.8)$ & $15(4.8)^{\star \star}$ \\
\hline
\end{tabular}

Abbreviations: PSG = progressive subcortical gliosis, PSP = progressive supranuclear palsy, WAIS-R = Wechsler Adult Intelligence Scale, Revised; FSIQ = Full Scale Intelligence Quotient $\mathrm{MQ}=$ Memory Quotient " Values represent mean (SD). Results of neuropsychological performance are available for only 17 of the normal controls.

performance are available for only 17 of the normal controls.
Significant differences between normals and all PSP subjects, definite and probable, using Student's two-tail $t$ test indicated by $\left.{ }^{\star \star}=\mathrm{p}<0.0001\right)^{\star}=\mathrm{p}<0.005,+=\mathrm{p}<0.05$. Values for cases Student's two-tail t test ind SD from the mean value in normal subjects are shown in bold.
$1-3$ that are more than 2 SD for
Five of the 14 patients we studied have subsequently died. In two necropsy could not be obtained. The other three have been studied postmortem with a standard protocol that includes gross and microscopic examination, including haematoxylin-eosin, phosphotungstic acid-haematoxylin, luxol fast blue-cresyl violet-eosin, and pyridine silver carbonate stains. In table 2 we have classified the 11 patients not examined at postmortem as having "probable" PSP.

\section{Results}

The clinical features of the three necropsy cases are summarised in table 1. Each of these individuals was observed over several years. At the time of their PET study all were given the clinical diagnosis of PSP, and this diagnosis remained unchanged throughout their illnesses. As we have previously reported, ${ }^{19}$ neuropsychological performance was significantly impaired in subjects with probable PSP compared with controls, and similar impairments were observed in all three patients at necropsy. This level of ability represented a decline from premorbid abilities ascertained by history in all three, but the patient with PSG had somewhat more severe impairments of memory relative to his intellectual abilities than the others at the time of his scan (table 2).

All of the patients had predominantly frontal hypometabolism in the cerebral cortex compared with normal controls of similar age (table 2). The three pathologically examined patients had average glucose metabolic rates in the cerebral cortex below the mean of normals and within the range of those observed in the probable PSP group. Metabolic rates in the frontal cortex relative to the rest of the cerebral cortex, and relative to posterior cortical regions were more than 2 standard deviations below that observed in the normal subjects (table 2). This was even more marked in the PSG patient than in the definite PSP patients. In these patients, even though only a few individual regions had metabolic rates that were more than 2 standard deviations from normal, almost all values were closer to those observed in the PSP subjects than in normals. All demonstrated a similar anterior to posterior gradient for metabolism that was like that seen in the probable PSP group (figure 1).

Despite these metabolic similarities, the patient with PSG had substantial neuronal loss and astrocytic gliosis in the frontal cortex, whereas no abnormalities were found in the cerebral cortex of patients with PSP (table 3). Metabolism of the caudate nucleus, putamen, pons, and thalamus in all three patients was below the mean of the normal controls and similar to that seen in the patients with probable PSP (table 2). The distribution of hypometabolism and pathology in subcortical structures do not always correspond and the severity of one does not necessarily reflect the severity of the other. This suggests that deafferentation may also play a role in subcortical hypometabolism.

CT scans in all three patients examined 


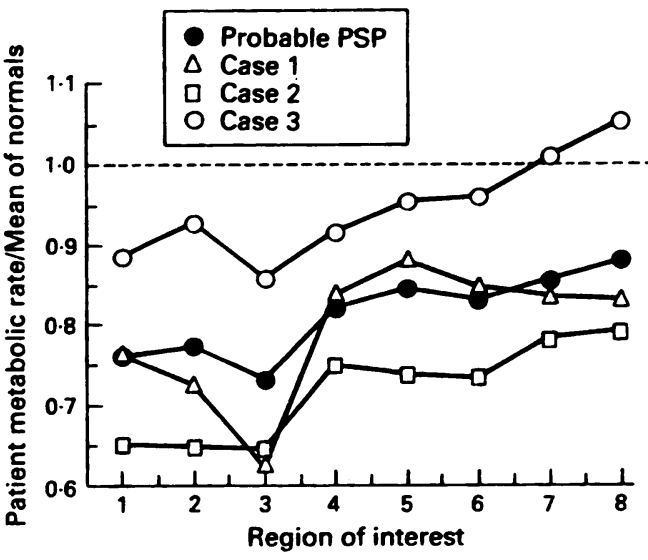

Figure 1 Regional cerebral cortical metabolic rates for glucose in patients with probable progressive supranuclear palsy (PSP) (values represent the mean of 11 subjects), pathologically confirmed PSP (Cases 2 and 3) and a patient with clinical features of PSP and pathological diagnosis of progressive subcortical gliosis (PSG) (Case 1), expressed as a ratio to the mean rate observed in the same region in normal subjects. Values for each region are the means of sectors in both hemispheres from each of 6 horizontal sections (12 sectors). Regions of interest extend from the most anterior (1) to the most posterior (8) part of the cerebral cortex. Similar patterns of metabolism are seen in all patients with metabolism relatively lower in more anterior regions. Metabolic rates observed in the patient with PSG fall within the range of values observed in patients with PSP.

pathologically were read as normal for age. The cerebral cortical sulci, lateral and third ventricles were larger in the patient with PSG than in those with definite PSP, but they otherwise did not differ and none demonstrated pontine or cerebellar atrophy.

The clinical course, symptoms, neurological and neuropathological findings are described for each patient:

\section{Progressive subcortical gliosis}

Case 1 This chemical engineer complained of blurred vision while reading in the spring of 1983 at the age of 57 . This progressed, and in the fall of the same year he developed difficulty keeping his eyelids open and turning his eyes. $\mathrm{He}$ also had hesitancy and monotony of speech without dysphagia. The following spring, loss of voluntary upgaze, vertical and horizontal

Table 3 Pathological findings in the cerebral cortex and subcortical brain regions in a patient with progressive subcortical gliosis and 2 patients with progressive supranuclear palsy

\begin{tabular}{|c|c|c|c|}
\hline Region & $\begin{array}{l}\text { Case } 1 \\
P S G\end{array}$ & $\begin{array}{l}\text { Case } 2 \\
P S P\end{array}$ & $\begin{array}{l}\text { Case } 3 \\
\text { PSP }\end{array}$ \\
\hline $\begin{array}{l}\text { Frontal cortex } \\
\text { Pyramidal tract in peduncles, pons, } \\
\text { medulla }\end{array}$ & $\underset{\mathrm{N}}{\mathrm{N}}, \mathrm{G}$ & $\begin{array}{l}(-) \\
(-)\end{array}$ & $\begin{array}{l}(-) \\
(-)\end{array}$ \\
\hline $\begin{array}{l}\text { Caudate nucleus } \\
\text { Putamen }\end{array}$ & $\begin{array}{l}\mathrm{N}, \mathrm{G} \\
\mathrm{N}, \mathrm{G}\end{array}$ & $\begin{array}{l}(-) \\
(-)\end{array}$ & $(-)$ \\
\hline Globus pallidus & $\begin{array}{l}N, G \\
N, G\end{array}$ & $\stackrel{(-)}{N}, G, T$ & $\mathrm{~N}, \mathrm{G}, \mathrm{T}$ \\
\hline Posterior medial thalamus & $\mathrm{G}$ & $(-)$ & G \\
\hline Ventral thalamus & $(-)$ & G & $(-)$ \\
\hline Subthalamic nucleus (Luys) & $(-)$ & G & G \\
\hline Substantia nigra & $\mathrm{N}, \mathrm{G}$ & $\mathrm{N}, \mathrm{G}$ & $\mathrm{N}, \mathrm{G}$ \\
\hline Midbrain tectum & $\mathrm{G}$ & $\mathrm{N}, \mathrm{G}, \mathrm{T}$ & $\mathrm{G}, \mathrm{T}$ \\
\hline Red nucleus & G & $\mathrm{G}, \mathrm{T}$ & $\vec{G}$ \\
\hline Pontine nuclei & $(-)$ & $(-)$ & $(-)$ \\
\hline Cerebellar dentate nucleus & $(-)$ & $\mathrm{N}, \mathrm{G}$ & $\mathrm{N}, \mathrm{G}$ \\
\hline $\begin{array}{l}\text { Inferior olive } \\
\text { XII nerve nucleus }\end{array}$ & $\begin{array}{l}\mathrm{N}, \mathrm{G} \\
\mathrm{N}, \mathrm{G}\end{array}$ & $\underset{(-)}{\mathbf{N}, \mathbf{G}}$ & $\underset{(-)}{\mathbf{N},} \mathbf{G}$ \\
\hline
\end{tabular}

Abbreviations: PSG = progressive subcartical gliosis, $\mathrm{PSP}=$ progressive supranuclear palsy, $\mathrm{N}=$ substantial loss of neuron perikarya or axons, $\mathrm{G}=$ astrocytic gliosis (hypertrophied astrocytes), $\mathrm{T}$ $=$ neurofibrillary tangles in neurons, $(-)=$ no abnormality. optokinetic nystagmus and convergence with preservation of reflex upgaze were observed and a diagnosis of PSP was considered. Later in 1984 he developed axial and extremity rigidity, emotional incontinence and progressive dementia leading to the loss of his job in April 1985. He was unresponsive to methylphenidate, pemoline, levodopa/carbidopa, bromocriptine, monoamine oxidase inhibitors and tricyclic antidepressants. He had a large prostate and developed urinary incontinence. There was no family history of movement disorders or dementia.

In August 1985, at the time of the PET study, neurological examination revealed normal visual acuity, small square wave jerks with fixation, absent optokinetic nystagmus and Bell's phenomenon, and jerky visual pursuit movements. Gaze was dysconjugate with $5^{\circ}$ of upgaze and now only $30^{\circ}$ of downgaze. Horizontal gaze was $70 \%$ of normal. The limitations in ocular movements were less marked with oculocephalic testing. His neck was held flexed and he demonstrated moderate bradykinesia and difficulty turning, but was able to get out of a chair unassisted using his hands. He had severely increased resistance to passive manipulation of the neck, more apparent on flexion than on extension, mild bifacial weakness, a decreased gag reflex, slowed and uncoordinated tongue movements, a moderately severe dysarthria with spastic and hypokinetic components, a brisk jaw jerk, moderate resistance to passive manipulation in all extremities without cogwheeling, and snout and glabellar reflexes. He scored below normal on all formal tests of neuropsychological ability with impaired learning of new materials, poor retention of previously learned information, substantial memory impairment for verbally and visually presented materials and especially low verbal fluency (table 2 ).

In the Autumn of 1985 he developed increased difficulty in swallowing and choked on food frequently. By the spring of 1986 his speech was unintelligible and he communicated by writing. He held his right arm and leg flexed and had bilateral Babinski signs. He began to fall frequently and suffered multiple rib fractures. In November 1986, he lost the ability to move his hands, and with this, his only surviving means of communication. During the last months of his life he developed chronic anaemia and decubitus ulcers, and despite placement of a gastrostomy tube, he had recurrent aspiration pneumonias from which he died in August 1987.

Pathology The brain weighed 1280 grams and showed moderate atrophy of the frontal lobes bilaterally (figure 2 ). On gross examination the temporal, parietal, and occipital lobes, cerebellum and brainstem were unremarkable. On microscopic examination, the sensorymotor, frontal and insular cortex showed extensive areas of diffuse neuronal loss with replacement by an isomorphic gliosis. The cortex demonstrated no neurofibrillary tangles, Pick bodies or neuritic plaques. The white matter underlying the sensory-motor cortex showed myelin pallor which could be traced 


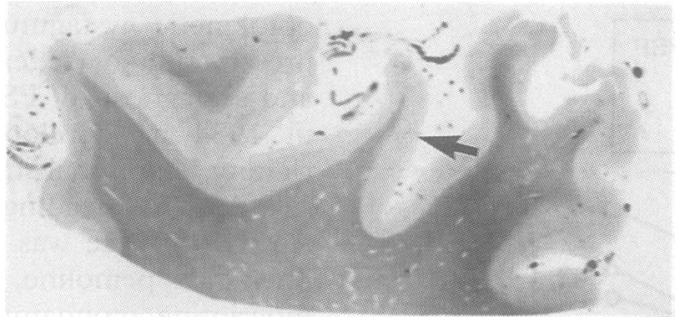

Figure 2 Frontal cortex from case 1, showing marked cortical atrophy with shrunken gyri (arrow). The pallor in subcortical white matter corresponds to areas of subcortical astrogliosis shown in figure 4. Magnification $\times 1 \cdot 5$.

LFB-cresyl violet-eosin stain.

throughout the corticospinal tracts in the internal capsule, the crus cerebri, pons and the pyramids of the medulla (figure 3). A striking finding in the subcortical white matter of the frontal, insular, and to a lesser extent temporal lobe was the presence of patchy areas of marked gemistocytic astrogliosis (figure 4). Similar relatively circumscribed areas of intense gliosis and neuronal loss with occasional gemistocytes were seen in the inferior colliculi, tegmental pons and the ventral inferior olivary nucleus. The globus pallidus, dorsal putamen and caudate nucleus showed moderate neuronal loss and isomorphic gliosis. The thalamus and the subthalamic nucleus were unremarkable. In particular, no neurofibrillary tangles were found in the subthalamic nucleus, globus pallidus or midbrain to substantiate the diagnosis of PSP. The hippocampus appeared normal. The substantia nigra showed a mild neuronal loss with extracellular pigment. The cerebellum was microscopically unremarkable.

\section{Definite PSP}

Case 2 In 1982 at the age of 58 this woman noted trouble finding words, recalling names, places, and past events, and developed slowness of walking, speaking and limb movements. She seemed stiff and poorly coordinated and fell occasionally, usually backward. Her symptoms slowly progressed so that by 1984 her handwriting had become small and poorly legible, and she had developed a tremor in the left arm and leg and incoordina-

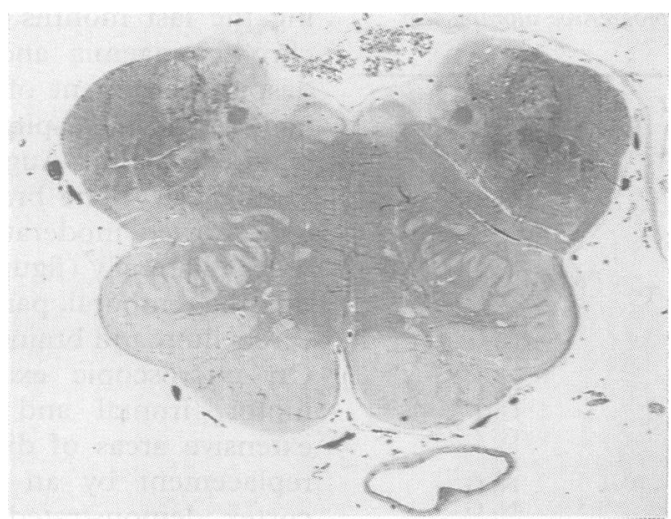

Figure 3 Medulla oblongata from case 1 demonstrating degeneration of the corticospinal tracts and the lower limbs of the inferior olivary nuclei. Magnification $\times 3 \cdot 2$. LFB-cresyl violet-eosin stain.

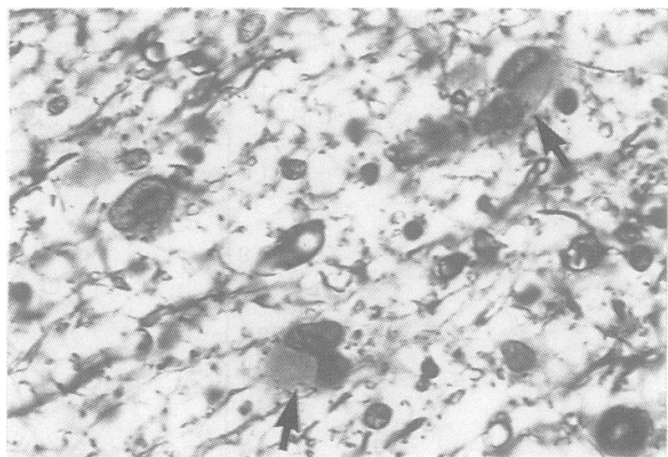

Figure 4 Micrograph of the subcortical white matter in case 1 showing clusters of large (gemistocytic) astrocytes (arrows) containing generous eosinophilic cytoplasm and large vesiculated nuclei. Magnification $\times 400 . P T A H$ stain.

tion of her right leg when walking. She also had difficulty chewing and swallowing, choking frequently. Her symptoms were not relieved by treatment with levodopa/carbidopa, bromocriptine, or lioresal.

In August 1986, when a PET study was performed, all of her symptoms had progressed so that she spoke infrequently, had increasing difficulty swallowing, walked poorly, and often fell. On examination she was fully oriented and had a good fund of knowledge, but was poor at calculation and abstraction. She needed assistance with the upper arms when rising from a chair. Her posture was stooped and she walked slowly with freezing and festination. Her balance was poor so that she would fall if not caught. Visual acuity, pupillary reflexes and visual fields were normal. There was an exophoria of five prism diopters and large amplitude square wave jerks during both near and distant fixation. Voluntary eye movements were limited to $80 \%$ of normal with horizontal gaze, $5 \%$ of normal in upgaze and $60 \%$ of normal in downgaze. Saccades were slow and hypometric, pursuit movements were saccadic, and optokinetic nystagmus was deficient with horizontally moving targets and absent with vertically moving targets. Oculocephalic reflexes and Bell's phenomenon were intact. The face showed poor expressiveness, and speech was moderately dysarthric, with spastic and hypokinetic components. The gag reflex was hyperactive, and glabellar, palmomental, and snout reflexes were present. There was marked rigidity of the neck and the right upper extremity, less rigidity in the left upper extremity, and tremor at rest in both upper extremities, worse in the left than the right. The reflexes were 3+ throughout and the plantar responses were flexor. On neuropsychological testing impaired function was noted on all cognitive tasks except for the retention of newly learned materials, which was relatively maintained (table 2).

During the remaining two years of her life, the patient had progressive difficulty with ambulation, requiring use of a walker by July 1987. Nocturnal urinary incontinence and constipation developed in late 1987 and her speech was reduced to monosyllables. By July 1988 , she was totally unable to control her urinary-bladder function, had difficulty with 


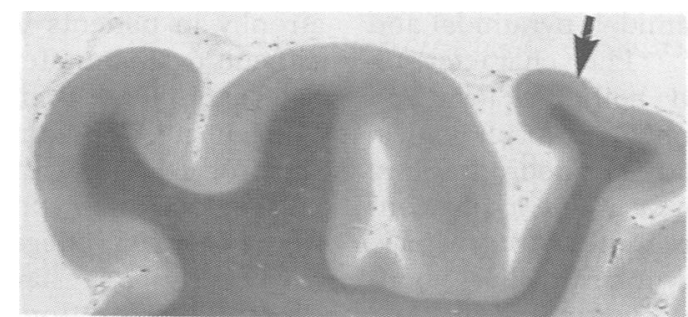

Figure 5 Frontal cortex from case 2 showing a normal cortical thickness and gyri (arrow). Magnification $\times 1 \cdot 5$. LFB-cresyl violet-eosin stain.

chewing and often choked. She died in a nursing home at the age of 64 .

Pathology The brain weighed 1150 grams and appeared normal on gross inspection without noticeable cortical atrophy. The cerebellum and brainstem appeared grossly unremarkable. On microscopic examination the frontal, temporal and occipital cortex appeared normal and no neurofibrillary tangles or neuritic plaques were seen (figure 5). An occasional tangle was found in the parahippocampal cortex. Moderate to marked neuronal loss with astrogliosis was seen in the anterior globus pallidus, anterior thalamus, subthalamic nucleus, inferior olivary nucleus, and dentate nucleus of the cerebellum. Occasional neurofibrillary tangles were demonstrated in the globus pallidus (figure 6), red nucleus and periaqueductal grey of the midbrain.

\section{Definite PSP}

Case 3 In 1984 at the age of 65 , this woman complained of progressive difficulty in reading because of blurring of vision. Soon thereafter her speech became slurred and she choked on solid foods. Writing became difficult and she was unable to recall recent events and conversations. She experienced falls nearly every day.

By the time of her PET study in April 1986 she had lost $25 \mathrm{lbs}$ in weight. She was fully oriented, had a good fund of information, intact naming, and abstract proverb interpretation. Her posture was stooped with a narrow based and mildly unsteady gait; turns required several steps. Visual acuity, pupillary reflexes and visual fields to confrontation were

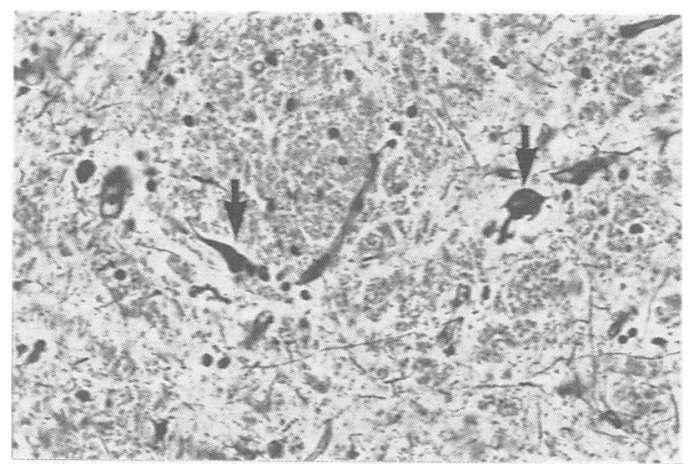

Figure 6 Globus pallidus from case 2 showing neurofibrillary tangles (arrows). Magnification $\times 400$. Hicks' pyridine silver stain. normal. Voluntary horizontal gaze was limited to $50 \%$ of normal, upgaze was $60 \%$ of normal and downgaze was $10 \%$ of normal. Pursuits were saccadic and saccadic movements were slowed with optokinetic nystagmus absent in all directions. Gaze was full with oculocephalic reflexes. There was a mild dysarthria with spastic and hypokinetic components and a hyperactive gag reflex. Facial expressiveness was decreased, and there was infrequent blinking. The deep tendon reflexes were brisk throughout and the plantar responses were downgoing. There was a snout reflex. Neuropsychological testing showed relatively well maintained general intellect and vocabulary, moderately impaired verbal fluency, and below average initial learning (table 2). Once learned, however, information obtained either verbally or visually was retained well.

The patient's difficulty with speech, swallowing, and walking continued, and she sustained several falls with fractured ribs and hips. Her difficulty with swallowing led to progressive loss of weight. A memory and behavioural disturbance became evident in April 1988 when she could not recall her daughter's name, and appeared to talk to individuals who were not in the room. She then became completely dependent for all her care. Her posture was markedly stooped and she needed assistance in arising from a chair and walking, since she took irregular steps and often fell unexpectedly. There was marked slowness of limb movements and moderate neck rigidity in extension, but rigidity was present in the extremities only upon activation. Horizontal gaze was limited to $50 \%$ of normal, upgaze to $10 \%$ of normal and downgaze was absent. Movements were uncoordinated and slow, and her writing had become illegible. In July 1988 she was admitted to hospital for a gastrostomy because of progressive inability to swallow. She died in a nursing home at the age 70 .

Pathology The brain weighed 1340 grams and was normal on gross examination. On microscopic examination the findings were similar to case 2 . The frontal, temporal and parietal cortex were normal and no neurofibrillary tangles or neuritic plaques were seen. There was neuronal loss with moderate astrogliosis in the globus pallidus, dorsal part of the amygdala, anterior thalamus, and dentate nucleus. Moderate neuronal loss and gliosis was noted in the inferior olivary nucleus. The substantia nigra showed moderate neuronal loss and gliosis. Neurofibrillary tangles were demonstrated in the globus pallidus and periaqueductal grey.

\section{Discussion}

PSP is characterised pathologically by localised neuronal loss, gliosis, and distinctive neurofibrillary tangles conspicuously affecting the globus pallidus, subthalamic nucleus, red nucleus, substantia nigra, dentate nucleus, tectum, and periaqueductal grey matter. The caudate nucleus and putamen are less affected and the cerebral cortex is generally spared..$^{2021}$ PSG is an entity with neuropathological 
changes within extrapyramidal, pyramidal and cerebellar systems. ${ }^{1217}$ The characteristic features are widespread neuronal loss and gliosis, but without distinctive morphological changes such as Lewy bodies or neurofibrillary tangles. These changes are variable in distribution from one patient to another, but usually involve the basal ganglia, inferior olives, pontine nuclei, cerebellum, Purkinje cells, and frequency the cerebral cortex. ${ }^{16}$

The clinical symptoms and signs of PSP and PSG can be similar. ${ }^{12}$ PSP causes supranuclear palsy of gaze, axial dystonia, pseudobulbar palsy with dysarthria and dysphagia, bradykinesia, rigidity, and a progressive dementia. ${ }^{2021}$ PSG causes a variety of symptoms and its clinical recognition is difficult since it can present with signs of disease affecting extrapyramidal, pyramidal, and cerebellar pathways, as well as dementia. Although it would be reassuring if a particular clinical feature could be relied upon to distinguish these two disorders, this is not possible because multiple subcortical structures are involved in both. Each of the symptoms exhibited in our patients has been reported previously in individuals with pathologically verified PSP or PSG. Abnormal eye movements, including voluntary upgaze and downgaze paresis, were seen in all of our subjects. While the loss of upgaze more than downgaze is not common in PSP, it cannot be considered a reliable sign of PSG, since it was also seen in one of our patients with PSP. Likewise, resting tremor cannot be used to identify these disorders, since it has been observed in patients with PSG, ${ }^{12}$ it was seen in one of our patients with PSP, and it is a typical feature of Parkinson's disease. The Babinski signs seen in our patient with PSG appeared only late in his illness and probably reflect the neuronal loss and gliosis observed in the cerebral cortex and degeneration of the corticospinal tracts. These signs of corticospinal tract disease cannot be used to distinguish PSG from PSP, since these signs, including Babinski reflexes, have also been observed in patients with pathologically confirmed PSP. ${ }^{21}$

Neuropsychological testing does not distinguish PSG from PSP subjects, but our results leave open the possibility that there may be some differences in the cognitive impairments in these two conditions. Memory and recall on tasks of new learning were substantially more impaired in our PSG patient than in either of the PSP patients. For example, subject 1 had a memory quotient 17 points lower than subject 2 even though their full scale intelligence scores were identical. As we have previously reported in patients with dementia, ${ }^{19}$ the degree of relative frontal hypometabolism in these patients paralleled the severity of their impairment of verbal fluency, a disturbance that is likely mediated by the frontal lobe.

Structural brain imaging also does not differentiate PSG from PSP. In our patient with PSG, cerebral atrophy appeared grossly to be more pronounced than in the two patients with PSP, but this is a non-specific finding. Both CT and MRI can show cerebral and brainstem atrophy in patients with PSP, ${ }^{22}{ }^{24}$ but this is difficult to quantitate, and was not apparent in our cases. Decreased T2 relaxation times can be seen in the putamen, globus pallidus, and superior colliculi with high field MRI, but this finding does not appear to differentiate PSP from other neurodegenerative disorders. ${ }^{24}$

FDG-PET may have utility in differentiating PSP and PSG from other conditions. Our findings confirm our previous observation that PSP causes predominantly frontal hypometabolism in the cerebral cortex and hypometabolism in several subcortical structures with relative sparing of the cerebellum ${ }^{2}$ and provide preliminary evidence that PSG can cause a similar abnormalities. This pattern is unlike the typical findings in patients with Parkinson's disease, Parkinson's disease with dementia, Alzheimer's disease, familial and sporadic olivopontocerebellar atrophy or normal control subjects. ${ }^{2525}$ Unfortunately, FDG-PET cannot reliably distinguish between PSP and PSG, as our patients illustrate, and clinically diagnosed striato-nigral degeneration also exhibits similar abnormalities. ${ }^{26}$

It is easier to demonstrate differences in metabolism between diagnostic groups, than to make diagnostic decisions in an individual. The similarities in the pattern of metabolism of Cases 1-3 to the PSP subjects lead the observer to classify them with the PSP cases, even though in all 3 only relative frontal hypometabolism is statistically different from normal controls. Although PSP patients as a group clearly have subcortical hypometabolism, in a particular individual this may not be apparent. Subcortical metabolic rates in the patients at necropsy were not more than 2 standard deviations below the mean of normals, but it can not be concluded that subcortical regions were unaffected. Subcortical metabolic rates in the necropsy patients were below the mean value of normal controls, and pathological abnormalities were later documented. Likewise, it is dangerous to draw conclusions about a diagnostic group from a single case. Although the pons was less hypometabolic in the patient with PSG than in PSP subjects, it is doubtful whether this is of any significance. PET will more dependably distinguish various multiple system degenerations in individual patients when FDG studies can be augmented by studies using more selective and specific ligands.

While the metabolic patterns of PSP and PSG may be similar, their origins in these two disorders appear to differ. Morphological changes in the cerebral cortex have been described occasionally in patients with PSP, ${ }^{27}$ and therefore the cause of the frontal hypometabolism seen in this condition with FDG-PET has been unresolved. However, since no pathological abnormalities were seen in the cerebral cortex of our patients with definite PSP, cerebral hypometabolism in these patients seems to be due to deafferentation alone, as was suggested previously. ${ }^{12}$ Conversely, in our patient with PSG it is likely that both deafferentation and intrinsic pathology account for the metabolic abnormalities in the 
cerebral cortex. Neuronal loss and gliosis in the cerebral cortex was not uniformly distributed and was most marked in the frontal cortex corresponding to the areas of greatest metabolic deficit. In both PSP and PSG, the subcortical hypometabolism appears to be a combination of intrinsic pathology and disconnection since no strict correlation between metabolism and pathology could be obtained.

This study was supported in part by NIH grants NS15655 and AG08671, and the Louise Madsen Fund. Dr Foster was recipient of a Clinical Investigator Development Award (NS01023) from the National Institute of Neurological Disorders and Stroke during part of this study. We thank Dr Bruno Giordani for his advice and assistance.

1 D'Antona R, Baron JC, Samson Y, et al. Subcortical dementia: frontal cortex hypometabolism detected by positron tomography in patients with progressive supranuclear palsy. Brain 1985;108:785-99.

2 Foster NL, Gilman S, Berent S, et al. Cerebral hypometabolism in progressive supranuclear palsy studied with (1988;24: 399-406.

3 Rougemont D, Baron JC, Collard P, et al. Local cerebral glucose utilisation in treated and untreated patients with Parkinson's disease. F Neurol Neurosurg Psychiatry 1984; 47:824-30.

4 Kuhl DE, Metter EJ, Riege WH. Patterns of local cerebral glucose utilization determined in Parkinson's disease by the (18F) fluorodeoxyglucose method. Ann Neurol 1984;15:419-24

5 Foster NL. Brain imaging as a useful tool in the diagnosis of dementia. In: Altman HJ, ed. Alzheimer's and Parkinson's diseases: recent advances in research and clinical management. New York: Plenum Press, 1989:19-38.

6 Boller F, Lopez OL, Moossy J. Diagnosis of dementia: clinicopathologic correlations. Neurology 1989;39:76-9.

7 Miller NR. Walsh and Hoyt's clinical neuro-ophthalmology, 4th ed. Baltimore: Williams and Wilkins, 1985:726-27.

8 Koeppen AH, Hans MB. Supranuclear ophthalmoplegia in olivopontocerebellar degeneration. Neurology 1976; 26:764-8.

9 Fearnley JM, Revesz T, Brooks DJ, et al. Diffuse Lewy body disease presenting with a supranuclear gaze palsy. $7 \mathrm{Neurol}$ Neurosurg Psychiatry 1991;54:159-61.
10 Bertoni JM, Label LS, Sackellares JC, Hicks SP. Supranuclear gaze palsy in familial Creutzfeldt-Jakob disease. Arch Neurol 1983;40:618-22.

11 Fink JK, Filling-Katz MR, Sokol J, et al. Clinical spectrum of Niemann-Pick disease type C. Neurology 1989;39: $1040-9$.

12 Will RG, Lees AJ, Gibb W, Barnard RO. A case of progressive subcortical gliosis presenting clinically as Steele-Richardson-Olszewski syndrome. $\mathcal{F}$ Neurol Neurosurg Psychiatry 1988;51:1224-7.

13 Dubinsky RM, Jankovic J. Progressive supranuclear palsy and a multi-infarct state. Neurology 1987;37:570-6.

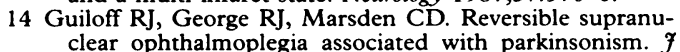
Neurol Neurosurg Psychiatry 1980;43:552-4.

15 Quinn N. Multiple system atrophy-the nature of the beast. 7 Neurol Neurosurg Psychiatry 1989;(Suppl):78-89.

16 Neumann MA, Cohn R. Progressive subcortical gliosis, a rare form of presenile dementia. Brain 1967;90:405-18.

17 Moossy J, Martinez AJ, Hanin I, et al. Thalamic and subcortical gliosis with dementia. Arch Neurol subcortical gliosis

18 Friedland RP, Budinger TF, Koss E, Ober BA. Alzheimer's disease: anterior-posterior and lateral hemispheric alterations in cortical glucose utilization. Neuroscience Letters 1985;53:235-40

19 Berent S, Foster NL, Gilman S, Hichwa R, Lehtinen S. Patterns of cortical ${ }^{18}$ F-FDG metabolism in Alzheimer's and progressive supranuclear palsy patients are related to the types of cognitive impairments. Neurology 1987;37(Suppl 1): 172 .

20 Steele JC, Richardson JC, Olszewski J. Progressive supranuclear palsy. Arch Neurol 1964;10:333-59.

21 Steele JC. Progressive supranuclear palsy. Brain 1972; 95:693-704.

22 Ambrosetto P, Michelucci R, Forti A, Tassinari CA. CT findings in progressive supranuclear palsy. $\mathcal{F}$ Comput Assist

23 Schonfeld SM, Golbe LI, Sage JI, et al. Computed tomographic findings in progressive supranuclear palsy: correlation with clinical grade. Movement Disorders $1987 ; 2: 263-78$

24 Drayer BP, Olanow W, Burger P, et al. Parkinson plus syndrome: diagnosis using high field $M R$ imaging of brain iron. Radiology 1986;159:493-8.

25 Gilman S, Markel DS, Koeppe RA, et al. Cerebellar and brainstem hypometabolism in olivopontocerebellar atrophy detected with positron emission tomography. Ann Neurol 1988;23:223-30.

26 DeVolder AG, Francart J, Laterre C, et al. Decreased glucose utilization in the striatum and frontal lobe in probable striatonigral degeneration. Ann Neurol 1989; 26:239-47.

27 Ishino H, Otsuki S. Frequency of Alzheimer's neurofibrillary tangles in the cerebral cortex in progressive supranuclear palsy (subcortical argyrophilic dystrophy). $\mathcal{f}$ Neurol Sci 1976;28:309-16. 